\title{
An Update on Poly(ADP-ribose) polymerase-1 (PARP-1) Inhibitors: Opportunities and Challenges in Cancer Therapy
}

\author{
Ying-Qing Wang ${ }^{\dagger, \S}$, Ping-Yuan Wang ${ }^{\ddagger}$, I,, Yu-Ting Wang ${ }^{\dagger}$, Guang-Fu \\ Yang, "Ao Zhang, , , and Ze-Hong Miao ${ }^{\dagger, *}$
}

${ }^{\dagger}$ Division of Antitumor Pharmacology, State Key Laboratory of Drug Research, Shanghai Institute of Materia Medica, Chinese Academy of Sciences, Shanghai 201203, China; ${ }^{\ddagger}$ CAS Key Laboratory of Receptor Research, and Synthetic Organic \& Medicinal Chemistry Laboratory, Shanghai Institute of Materia Medica, Chinese Academy of Sciences, Shanghai 201203; "Key Laboratory of Pesticide \& Chemical Biology, Ministry of Education, College of Chemistry, Central China Normal University, Wuhan 430079, China.

${ }^{\S}$ These authors contributed equally to this work.

"To whom correspondence should be addressed. For Z.H.M.: phone: +86-21-50806820; fax: 86-21-50806820; E-mail: zhmiao@simm.ac.cn. For A.Z.: phone: +86-21-50806035; fax: 86-21-50806035; E-mail: aozhang@ @imm.ac.cn.

\section{Supporting Information}

\section{Contents:}

1. Supporting Table 1 
Supporting Table 1. Olaparib (2) in combinational studies completed.

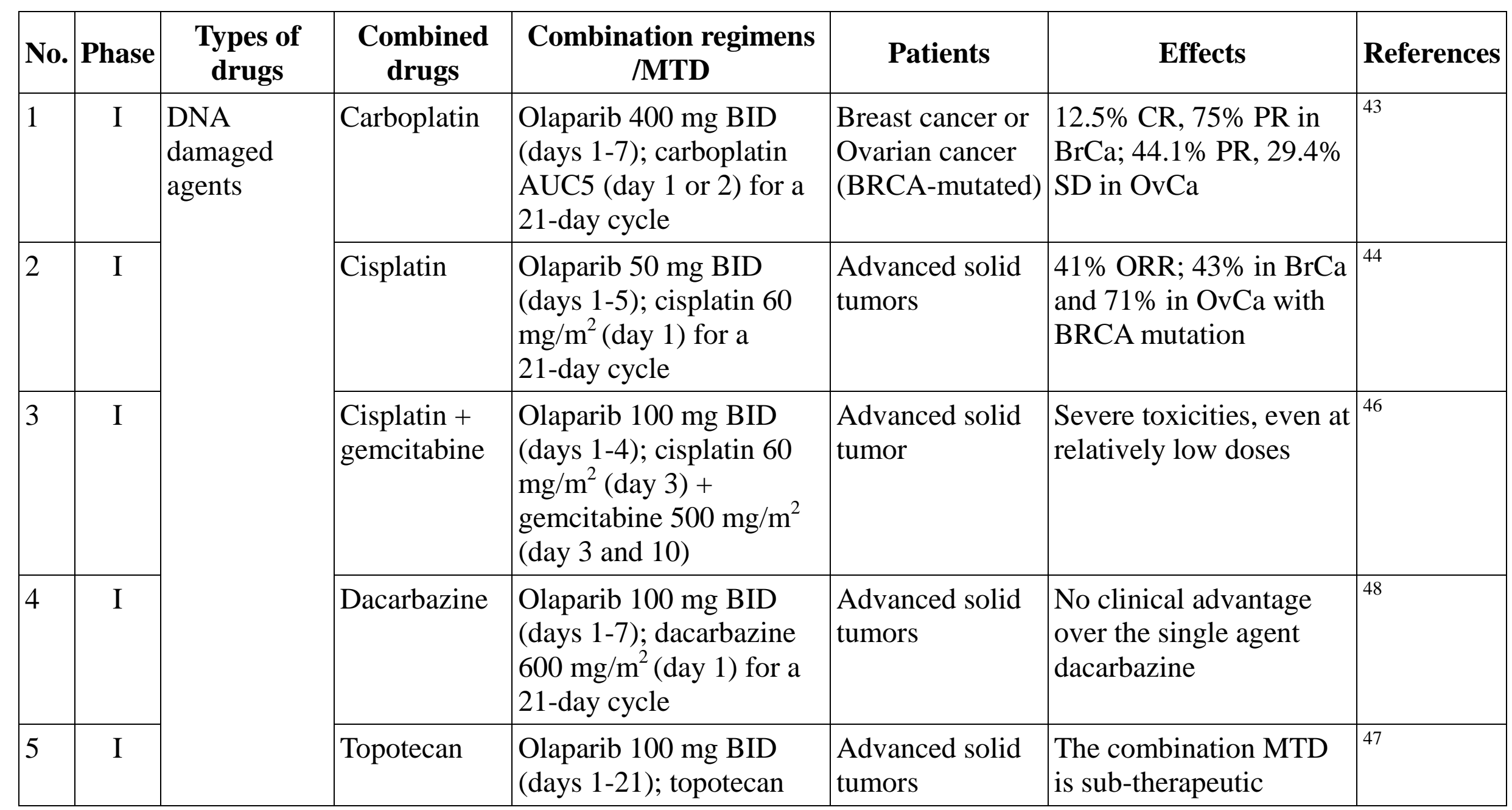




\begin{tabular}{|c|c|c|c|c|c|c|c|}
\hline & & & & $\begin{array}{l}1.0 \mathrm{mg} / \mathrm{m}^{2} / \text { day (days } 1-3 \text { ) } \\
\text { for a } 21 \text {-day cycle }\end{array}$ & & & \\
\hline 6 & I & & PLD & $\begin{array}{l}\text { Olaparib } 400 \mathrm{mg} \text { BID } \\
\text { (days 1-28); PLD } 40 \\
\mathrm{mg} / \mathrm{m}^{2} \text { (day 1) for a } \\
\text { 28-day cycle }\end{array}$ & $\begin{array}{l}\text { Advanced solid } \\
\text { tumors }\end{array}$ & $\begin{array}{l}33 \% \text { ORR, } 12 \% \mathrm{CR}, \\
29.4 \% \text { PR in OvCa; } 61 \% \\
\text { ORR in OvCa with } \\
\text { BRCA mutation }\end{array}$ & 45 \\
\hline 7 & $\mathrm{I}$ & \multirow{3}{*}{$\begin{array}{l}\text { Microtubule } \\
\text { inhibitors }\end{array}$} & Paclitaxel & $\begin{array}{l}\text { Olaparib } 200 \text { mg BID } \\
\text { (days 1-28); paclitaxel } 65 \\
\left.\mathrm{mg} / \mathrm{m}^{2} \text { (days } 1,8 \text { and } 15\right) \\
\text { for a } 28 \text {-day cycle }\end{array}$ & TNBC & $\begin{array}{l}37 \% \text { ORR. Optimized } \\
\text { schedule and dosing } \\
\text { strategy of olaparib and } \\
\text { paclitaxel are suitable } \\
\text { for TNBC treatment }\end{array}$ & \begin{tabular}{|l|l}
49 \\
\end{tabular} \\
\hline 8 & I & & $\begin{array}{l}\text { Paclitaxel + } \\
\text { carboplatin }\end{array}$ & $\begin{array}{l}\text { Olaparib } 150 \mathrm{mg} \text { BID } \\
\text { (days } 1-3 \text { each week); } \\
\text { paclitaxel } 60 \mathrm{mg} / \mathrm{m}^{2} \\
\text { (metronomic) + } \\
\text { carboplatin AUC2 } \\
\text { weekly in } 3 \text { weeks for a } \\
\text { 28-day cycle }\end{array}$ & Ovarian cancer & $\begin{array}{l}28.6 \% \text { CR; } 21.4 \% \text { PR; } \\
\text { no grade } 4 \text { toxicity } \\
\text { shown }\end{array}$ & 50 \\
\hline 9 & II & & $\begin{array}{l}\text { Paclitaxel + } \\
\text { carboplatin }\end{array}$ & $\begin{array}{l}\text { Olaparib } 200 \mathrm{mg} \text { BID } \\
\text { (days } 1-10 \text { ); paclitaxel } \\
\left.175 \mathrm{mg} / \mathrm{m}^{2} \text { (day } 1\right) ; \\
\text { carboplatin AUC4 (day } \\
\text { 1) for a } 21 \text {-day cycle }\end{array}$ & $\begin{array}{l}\text { Serous ovarian } \\
\text { cancer }\end{array}$ & $\begin{array}{l}\text { Improved PFS } \\
\text { significantly (12.2 vs. } \\
9.6 \text { months) }\end{array}$ & 51 \\
\hline
\end{tabular}




\begin{tabular}{|c|c|c|c|c|c|c|c|}
\hline & & & & $\begin{array}{l}\text { After } 6 \text { cycles, olaparib } \\
400 \mathrm{mg} \text { BID }\end{array}$ & & & \\
\hline 10 & II & & Paclitaxel & $\begin{array}{l}\text { Olaparib } 100 \text { mg BID } \\
\text { (days } 1-28) ; \text { paclitaxel } 80 \\
\text { mg/m² (days } 1,8 \text { and } 15) \\
\text { for a } 28 \text {-day cycle }\end{array}$ & Gastric cancer & $\begin{array}{l}\text { Significant OS but no } \\
\text { PFS improvement } \\
\text { versus paclitaxel (13.1 } \\
\text { vs. } 8.3 \text { months), } \\
\text { especially in ATM } \\
\text { patients }\end{array}$ & 52 \\
\hline 11 & I & \multirow{3}{*}{$\begin{array}{l}\text { Angiogenesis } \\
\text { inhibitors }\end{array}$} & Bevacizumab & $\begin{array}{l}\text { Olaparib } 400 \mathrm{mg} \text { BID; } \\
\text { bevacizumab } 10 \mathrm{mg} / \mathrm{kg} \\
\text { q2w }\end{array}$ & $\begin{array}{l}\text { Advanced solid } \\
\text { tumors }\end{array}$ & Well tolerated & 53 \\
\hline 12 & I & & Cediranib & $\begin{array}{l}\text { Olaparib } 200 \mathrm{mg} \text { BID; } \\
\text { cediranib } 30 \mathrm{mg} \text { qd }\end{array}$ & $\begin{array}{l}\text { TNBC or } \\
\text { Ovarian cancer }\end{array}$ & $\begin{array}{l}44 \% \text { ORR, } 61 \% \text { CBR in } \\
\text { OvCa; no response in } \\
\text { TNBC }\end{array}$ & 54 \\
\hline 13 & II & & Cediranib & $\begin{array}{l}\text { Olaparib } 200 \mathrm{mg} \text { BID; } \\
\text { cediranib } 30 \mathrm{mg} \text { qd }\end{array}$ & Ovarian cancer & $\begin{array}{l}\text { Improved PFS } \\
\text { significantly (17.7 vs } 9.0 \\
\text { months) }\end{array}$ & 55 \\
\hline 14 & I & \multirow{2}{*}{$\begin{array}{l}\text { Kinase } \\
\text { inhibitors }\end{array}$} & BKM120 & $\begin{array}{l}\text { Olaparib 50-200 mg } \\
\text { BID; BKM120 40-50 mg } \\
\text { for a 28-day cycle }\end{array}$ & $\begin{array}{l}\text { Serous ovarian } \\
\text { cancer or TNBC }\end{array}$ & $\begin{array}{l}\text { Showed clinical benefit } \\
\text { without detailed data }\end{array}$ & 56 \\
\hline 15 & $\mathrm{I}$ & & Gefitinib & $\begin{array}{l}\text { Olaparib } 200 \mathrm{mg} \text { tds; } \\
\text { gefitinib } 250 \mathrm{mg} \text { qd for a } \\
\text { 28-day cycle }\end{array}$ & $\begin{array}{l}\text { EGFR mutated } \\
\text { NSCLC }\end{array}$ & $\begin{array}{l}\text { Showed tolerability and } \\
\text { evidence of clinical } \\
\text { benefit }\end{array}$ & 57 \\
\hline
\end{tabular}


